\title{
Blockchain for automotive: An insight towards the IPFS blockchain-based auto insurance sector
}

\author{
Nishara Nizamuddin ${ }^{1}$, Ahed Abugabah ${ }^{2}$ \\ ${ }^{1}$ College of Technological Innovation, Zayed University, Dubai, United Arab Emirates \\ ${ }^{2}$ College of Technological Innovation, Zayed University, Abu Dhabi, United Arab Emirates
}

\begin{tabular}{l}
\hline Article Info \\
\hline Article history: \\
Received Aug 19, 2020 \\
Revised Dec 30, 2020 \\
Accepted Jan 12, 2021 \\
\hline
\end{tabular}

Keywords:

Blockchain

Decentralized auto-insurance

sector

Decentralized autonomous

vehicle

Ethereum

Interplanetary file system

Peer-to-peer networks

Smart contracts

\section{Corresponding Author:}

Nishara Nizamuddin

College of Technological Innovation

Zayed University

Dubai Academic City, Dubai, United Arab Emirates

Email: nishara.nizam@gmail.com

\begin{abstract}
The advancing technology and industrial revolution have taken the automotive industry by storm in recent times. The auto sector's constantly growing demand has paved the way for the automobile sector to embrace new technologies and disruptive innovations. The multi-trillion dollar, complex auto insurance sector is still stuck in the regulations of the past. Most of the customers still contact the insurance company by phone to buy new policies and process existing insurance claims. The customers still face the risk of fraudulent online brokers, as policies are mostly signed and processed on papers which often require human supervision, with a risk of error. The insurance sector faces a threat of failure due to losing and misconception of policies and information. We present a decentralized IPFS and blockchain-based framework for the auto insurance sector that regulates the activities in terms of insurance claims for automobiles and automates payments. This article also discusses how blockchain technology's features can be useful for the decentralized autonomous vehicle's ecosystem.
\end{abstract}

This is an open access article under the CC BY-SA license.

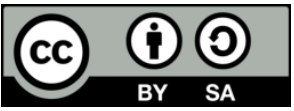

\section{INTRODUCTION}

The insurance sector is a conservative, highly centralized, multi-billion dollar business that has spanned across several industries including real estate, automobile industry and healthcare. The conventional model of the auto insurance sector has been one of the successful and reliable infrastructures since the time of its initiation. However, in recent times, the conventional auto-insurance sector has started to experience the change that modern technologies have brought into the insurance sector, concerning service and product delivery [1]. With technology influencing every industry, there is an increasing threat of cybersecurity attacks, denial of service attacks, and other IT security breaches that affect the digitalized automobile insurance industry leading to detrimental financial impacts [1-3]. Researchers and industry experts are constantly looking to identify new and to improve existing solutions to revolutionize the conventional automotive sector by incorporating innovative technologies to improve data collection, data management, integrity, security, and overall infrastructure of the automotive insurance sector [3]. New generation systems are being designed that are combined with data processing and a substantial amount of data management capabilities to communicate with humans with the minimal intervention [4]. An estimated amount of $\$ 169$ billion is planned to be allocated for implementing new technologies to improvise the automotive sector [5]. The insurance industry has a stable position and is dependable in industrialized and mature societies. 
However, in developing economies this sector faces serious problems due to economic, social, and political systems existing in those countries. Some of the biggest challenges faced by the insurance industry-lack of trust, mismanagement of insurance records, pricing problem, competition and regulations, conflicts of interest, settlement disputes, and lack of transparency [6-10]. The auto insurance sector is one of the important sectors that play a major role in the automotive industry, as it is vital in providing financial aid in case of an accident or damage caused due to an accident, theft, fire, natural disaster, or vandalism [11, 12]. But in case of an existing conventional insurance settlement process, whenever the clients submit an insurance claim, they expect the insurance company to pay and settle their claims at the earliest. The insurance company generally follows a pattern with predefined protocols and regulations, by coordinating with the partnered garages, to settle the insurance claims raised by the insured [13-15]. Acko general insurance limited [15], and existing literature for car and bike insurance, identifies five major issues faced by the auto-insurance sector when settling claims. The common challenges are identified to be:

- Absence of first information report at times of accident, theft, or vandalism which can be used for verifying the credibility of the claim submitted [15].

- Absence of insurance documents that shows that the validity of insurance policy $[15,16]$.

- Postponed claim-insured not acting immediately to apply for claims $[15,16]$.

- Lack of clear understanding of insurance company's policies and regulations by the consumers $[15,17,18]$.

- Consumer's lack of knowledge on inclusions and exclusions of auto-insurance coverage [15, 16].

- Identity theft, identity fraud, fraudulent transactions, or sensitive consumer data breach issues [15, 19-21].

Further, several research questions need to be addressed on the evolving autonomous transportation systems, its impact on the society, and its infrastructure [17, 22]. There is a need for a trustworthy, decentralized, and transparent framework to emphasize the insurance policies to the policyholders, to impose the regulations of the insurance policy, to regulate the transactions, and manage the entity accountability. Blockchain technology has gained immense popularity in recent times due to its ability to manage transactions in a tamper-proof, immutable, and transparent manner and has been incorporated into various industries including smart buildings, agriculture, healthcare, energy sector, banking and finance, the insurance industry, e-government services [23-27]. The automotive insurance industry is entering into the new era of information management and digitization, which when combined with blockchain technology can significantly improve how the policies are designed and insurance claims are verified. Blockchain is powered by smart contracts in which policies, terms and conditions, and insurance amount to be paid by the company could be finalized and verified. Hence, whenever a claim is made by the policy owner, the data stored on the blockchain can immediately be accessed and a decision can be made by the insurance company to instantly approve or reject any claims made by the policy owner. But blockchain may not be efficient enough in storing large records or data. In such cases where a need arises to store large files, the decentralized IPFS will help in storing the files and the hash of the file stored in the IPFS can be stored efficiently within the smart contract. All the parties of the network will be notified in case of any change in the document and the IPFS hash remains unique for a document. If the file gets corrupted, or modified the hash changes and the smart contract sends a notification to all parties, and calls off the transaction, thereby safeguarding the legitimate content of the document. In case, if a false claim is made by the insurance policyholder, or if the insurance company disagrees to pay the claim based on the contractual terms, the smart contract will immediately notify all the entities of the network and solves the dispute by paying the payments to the policyholder thus enhancing trust on the system and assuring transparency in transactions. This research paper's contribution can be summed up as:

- To provide an insight into various functional areas of vehicular ecosystem that can harness the features of blockchain technology.

- To carry out a study to identify potential opportunities for transforming the conventional automotive insurance sector by deploying blockchain technology.

- To propose an IPFS blockchain-based framework for the vehicle insurance sector, powered by Ethereum smart contracts to carry out the insurance claims process in a transparent and tamper-proof manner.

The remainder of the paper is composed as: Section 2 discusses a brief background on blockchain and the challenges faced by the conventional automotive industry. The proposed framework of the decentralized auto insurance sector is presented in section 3. Challenges faced in implementing blockchain technology for the automobile ecosystem is presented in section 4 . Section 5 concludes the paper.

\section{BACKGROUND}

In this section, we focus on providing a brief overview of existing technologies for vehicle insurance management, an overview of the overall vehicular ecosystem, and an insight into how blockchain technology 
can transform the automotive insurance management process. We also discuss some of the existing literature work that highlights different technologies used in the automotive sector to improve secure data management, elevate security, and improve consumer satisfaction. The automotive sector is a multi-billion dollar business that comprises various divisions including design, development, manufacturing, sales and marketing, maintenance, and managing insurance of vehicles $[5,16]$. The auto-insurance sector, on the other hand, is a multiparty, multi-billion dollar business that supports the vehicle owner when the automobile gets damaged due to an accident or natural calamity, stolen, or gets damaged due to the collision with other vehicles [11, 13, 28], and insurance policies, rules, and regulations differ according to regional policies [28, 29]. With the recent enhancement in technology and innovation in the auto sector with the booming up of electric vehicles, self-driving cars, and smart vehicles, automobile industries are looking forward to emerging technologies for effective and safe data management [30]. AIG [31], a business consulting firm, identifies the fact that the insurance industry's environment is constantly changing due to the introduction of autonomous functions and highlights the challenges involved in the ability of the industry's adoption towards rapidly changing data models. However, the report does not specify any particular technology but describes the adoption of new technologies in a generalized fashion [31]. PwC, USA emphasizes the business model in which organizations are ready in adopting new technologies and is focusing more on consumer satisfaction; and has identified the major factor that, small investments made in commercial insurance largely influence the success of any insurance sector [32].

Demir et al. [30] propose a blockchain-based vehicle insurance management system, in which vehicle insurance distributed ledger has been implemented to exchange details of insurance policies among stakeholders in the network. The authors [30] present the system which is transparent and is tamper-proof concerning- "proof-of-insurance", to all participating active nodes. The federal government of UAE and the road transport authority (RTA of Dubai) has launched a project named Dubai vehicle chain [33], which utilizes blockchain technology for vehicle lifecycle management. This system was built aiming to be a part of the Emirates blockchain strategy 2021 and Dubai blockchain strategy which is proposed to be completed by the year 2020 to handle vehicle registration, insurance management, and ownership of vehicles. On the other hand, Belgium's KBC bank [34] created a digital ecosystem to connect to all entities while purchasing a car with the influence of blockchain technology, to create a tamper-proof and trustworthy framework, thereby reducing hassles while purchasing an automobile. This will guarantee the authenticity of documents [34]. Various use cases of decentralized blockchain technology in the automobile industry are presented in Figure 1. IPFS [35], a decentralized file system could be used to hold the records of insurance companies in an accurate way, which includes maintenance, performance, and assisting drivers. Coordinating various activities including document management, propriety to access claims, verification of documents can be effectively performed with blockchain technology [33, 34].

Thomson Reuters [36] reports the fact that the insurance industry is overwhelmed with the fraud problems which is costing the industry about $\$ 40$ billion per annum. Various forms of auto insurance frauds have been identified by the FBI concerning insurance frauds, such as orchestrated accidents, misrepresented claims by the policyholders, falsification of identification records for claims, and many more [37-39]. Blockchain aims to solve the issue of identity theft and fraud [23], and several tamper-proof models were developed to improve the overall architectural security for better data management, communication within the network, credibility of data shared among the peers, and decentralized payment models for the automotive sector [23, 24, 30, 40-43]. A permissioned blockchain-based system for auto-insurance claims for traversing data and entity interaction was designed [44], that showed increased reliability, based on proof-ofwork executed and is verified by actively participating entities within the network. The study proved to be effective in reducing the processing time for the transactions with minimum overhead time, as it is the commonly faced issue when a distributed ledger is employed.

Ridesharing, rental cars, and carpooling have become a new way of transportation as presented by PwC and within the next years, around $40 \%$ of vehicles across Europe and Asia are predicted to adapt to resource sharing-based and cost-efficient auto service industry [45]. As the concept of pay-as-you-go models are advancing with the increasing demand from the consumers, there increased a need to develop a secure, transparent system to support drivers during the payment of insurance premiums that use rental cars. A blockchain-based solution for management and analysis of data and micro-insurance records was presented by Vo et al. [46] that regulates the pay-as-you-go insurance premium models and makes the transactions very transparent and notifies all the parties (including the insurance company, drivers, associated financial organization) about various events within the network. This model will equally beneficial for all parties involved concerning data management, data security, intra-network communication, and insurance premium handling. On the other hand, Chekriy et al. [47] reiterate that the deployment of blockchain technology in the auto insurance sector would reduce the occurrence of the error in assessing the damage of vehicles, premium calculation, and settlement of claims and thereby results in reduced overall costs for the insurance sector. Chekriy 
et al. [47] also emphasize that the IBM Watson IoT platform could be integrated with blockchain to improve the engagement of entities within the network that aims to result in better estimation of damage in case of an accident, and beneficial settlement for the insured vehicle.

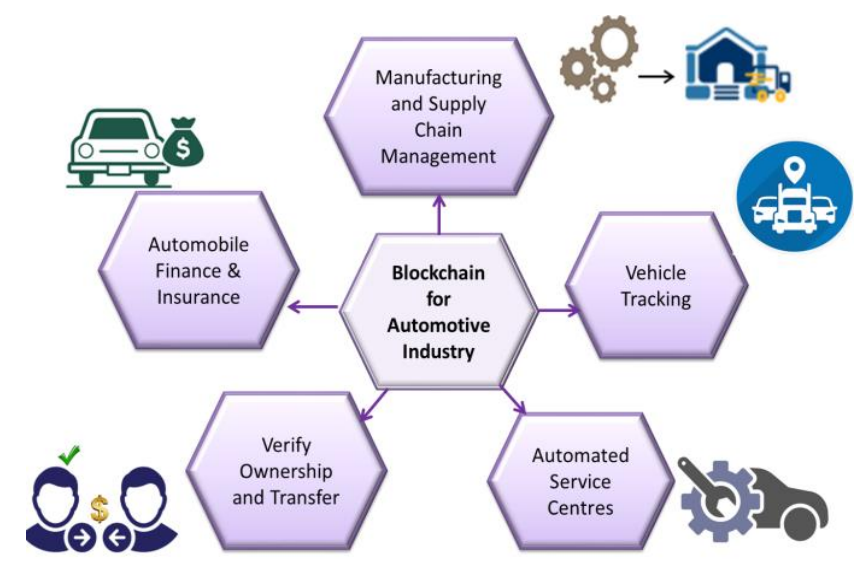

Figure 1. Blockchain use cases for automotive [23, 40, 44, 48]

\subsection{Blockchain use cases for vehicular ecosystem}

In this section, we list out various blockchain-based use cases for the vehicle ecosystem. We give an insight into several practical use cases ranging from financing and insurance applications, verifying ownership, manufacturing and supply chain management, new ownership models, and auto manufacturing. Table 1 presents the most predominant sectors in the auto sector where blockchain can play a major role in transforming the conventional auto sector.

Table 1. Blockchain-based use cases for the auto sector

\begin{tabular}{|c|c|}
\hline Blockchain Use cases for the Auto sector & Reference in Existing Literature/Industry \\
\hline Vehicle registration & Digital UAE [33]; Vo et al. [46]; European Start-up Prize for mobility [49] \\
\hline Ridesharing and new ownership models & $\begin{array}{c}\text { Demir et al. [30]; Viereckl et al. [45]; Vo et al. [46]; Chekriy et al. [47]; European Start- } \\
\text { up Prize for Mobility [49] }\end{array}$ \\
\hline $\begin{array}{l}\text { Interaction with external agents for } \\
\text { automobile maintenance and service }\end{array}$ & $\begin{array}{c}\text { Demir } \text { et al. [30]; E \& T [50]; Weerasinghe } \text { et al. [51]; European Start-up Prize for } \\
\text { mobility [49] }\end{array}$ \\
\hline Technology Innovations for auto-sector & $\begin{array}{c}\text { Digital UAE [33]; Daley [19]; Li et al. [42]; Gao et al. [43]; Yang et al. [41]; E \& T [50]; } \\
\text { Weerasinghe } \text { et al. [51]; Song et al. [52]; Stanciu [53]; Jensen } \text { et al. [54] }\end{array}$ \\
\hline Verification and transfer of ownership & $\begin{array}{c}\text { Fraga-Lamas } \text { et al. [48]; E \& T [50]; Gatteschi et al [55]; European Start-up Prize for } \\
\text { Mobility [49] }\end{array}$ \\
\hline Automobile financing & $\begin{array}{l}\text { Digital UAE [33]; Roy [34]; Gao et al. [43]; Oham et al. [44]; Vo et al. [46]; Chekriy } \\
\text { et al. [47]; Swan [10] }\end{array}$ \\
\hline $\begin{array}{l}\text { Blockchain and software-defined } \\
\text { approach for vehicular communities }\end{array}$ & $\begin{array}{c}\text { Yang et al. [41]; Gao et al. [43]; Yahiatene et al. [56]; Su et al. [57]; Aggarwal et al. [58]; } \\
\text { Aujla et al. [59] }\end{array}$ \\
\hline $\begin{array}{l}\text { Auto manufacturing and supply chain } \\
\text { management }\end{array}$ & $\begin{array}{l}\text { IBM [60]; Montecchi et al. [61]; Banerjee [62]; Ajao [63]; Chen et al. [13]; Abeyratne } \\
\text { et al. [64]; Song et al. [52]; Reimers et al. [65] }\end{array}$ \\
\hline Connected v & Balasubramaniam et al. [66]; Cebe et al. [67]; Stanciu [53]; Turato et al. [68] \\
\hline Decentralized vehicle insurance & $\begin{array}{l}\text { Dorri et al. [40]; Oham et al. [44]; Daley [19]; Demir et al. [30]; Vo et al. [46]; Chekriy, } \\
\text { et al. [47] }\end{array}$ \\
\hline $\mathrm{F}$ & Sharma et al. [69]; West [70] \\
\hline Trans: & Brousmiche et al. [71]; Ebrahim \\
\hline $\begin{array}{l}\text { Cost-effectiveness measures during a } \\
\text { product recall }\end{array}$ & $\begin{array}{c}\text { Reimers et al. [65]; IBM [60]; Montecchi et al. [61]; Banerjee [62]; Ajao [63]; Chen et al. } \\
\text { [23]; Abeyratne et al. [64]; Song et al. [52]; Swan [10] }\end{array}$ \\
\hline
\end{tabular}

\section{Problem statement:}

The conventional auto-insurance sector has been facing several challenges including verified insurance documents, manipulation of insurance policies, and consumer's lack of knowledge about the autoinsurance regulations, identity management, and insurance fraud due to data breach. There is a need for a trustworthy, tamper-proof, impartial regulatory framework to ensure the monitoring of all transactions transparently. 
A blockchain-IPFS based solution is proposed in the next section to ensure secure, transparent, and tamper-proof vehicle insurance transactions. Further, this framework aims to improve quality and productivity via automation, security, control, and traceability of insurance documents; Improved transparency among stakeholders gives a comprehensive view of data and transaction; Reinventing services through decentralized digital identities.

\section{PROPOSED SYSTEM-IPFS BLOCKCHAIN-BASED SOLUTION FOR THE VEHICLE INSURANCE SECTOR}

In this section, we propose a blockchain-powered solution that helps in regulating the vehicle insurance sector's functioning, claims management, and payment regulation and claim approvals. Figure 2 shows the proposed blockchain-based model for improving the existing vehicle insurance sector and automatically channelizing vehicle insurance claims. As shown in Figure 2, the actors/entities in the system are policyholder (customer), vehicle insurance company, garage representative, and Police department.

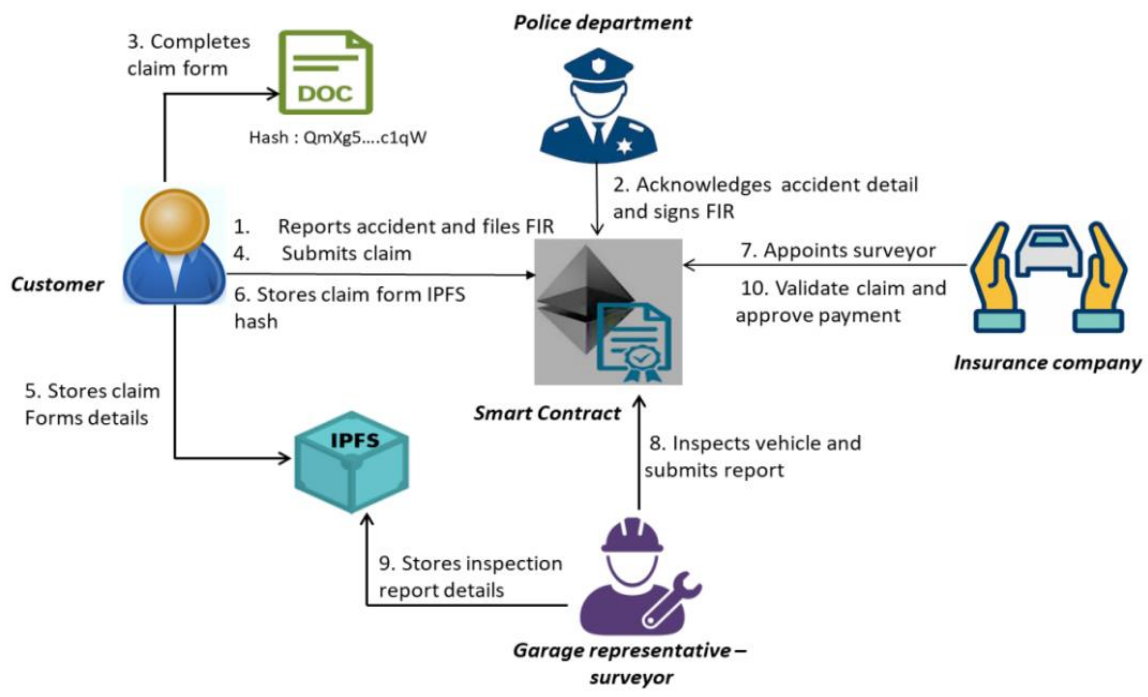

Figure 2. Blockchain-powered vehicle insurance sector

There is an Ethereum powered smart contract which controls and regulates all the entities involved in the insurance claim process. Initially, upon an accident or theft, the customer reports the incident and files the FIR. This is logged as an event in the smart contract. Following which the police department investigates and endorses the FIR registered by the consumer or insured in this case. As the next step, it is clear from Figure 2 that the customer completed and submits the claim form. The smart contract sends out an event to notify all the active participants about every function executed within the contract.

The customer then stores the claim form in the interplanetary file systems (IPFS), the decentralized file system that supports the smart contract for file storage purposes. A smart contract is not efficient enough to store large documents; hence the reports or documents are stored on the IPFS file system and IPFShash of the document or claims form in this case is stored on the smart contract. In case, if the report/document is modified or corrupted by a malicious user, the hash changes showing that the file was changed and the transaction gets aborted. The insurance company appoints a garage surveyor to inspect the vehicle and to validate the claim submitted by the customer. Once the garage surveyor validates the claims, the payment process is carried out and is paid to incur the expenses caused due to the accident. If the garage personnel deny the amount of claim submitted, then the contract denies payment for the claim and sends out an event notifying the concerned customer.

Figure 3 shows the sequence diagram explaining various functions and events between the participating entities. Using blockchain and smart contracts regulates and automates the communication between multiple parties in a network, and eliminates the need for middlemen to settle disputes in insurance claims. Upon raising the service request, the smart contract executed will guarantee the enforcement of predefined rules such that all the authorized stakeholders can check the outcome of a transaction. The proposed model aims to optimize the performance of the auto-insurance industry, making the transactions transparent and tamper-proof, and effective in terms of dispute settlement among parties. 




Figure 3. Sequence diagram showing a decentralized insurance claim process

\subsection{Implementation}

In this section, we have provided some of the important code snippets, and algorithms of our proposed solution, explaining the working principle of our smart contract. Algorithms 1 and 2 explain the insurance claim working principle on a blockchain platform powered by Ethereum smart contracts with IPFS decentralized file storage facility.
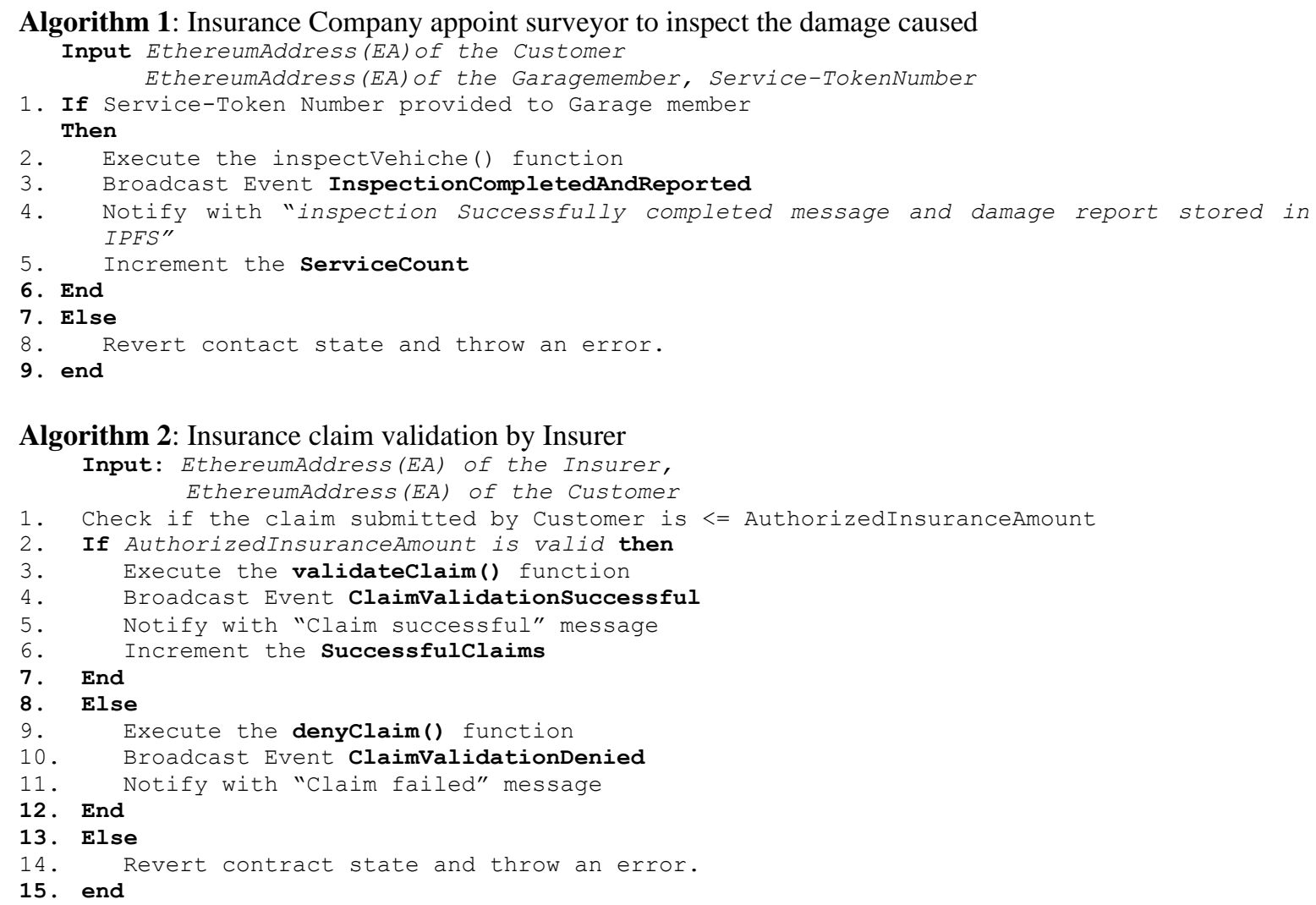

Algorithm 1 shows the smart code pseudo-code for the inspection carried out by the garage surveyor, in which the Ethereum addresses of the customer, garage member, and service token number is taken as input, as provided by the Insurance company. Upon receiving the assigned service token number, the garage member inspects the vehicle and reports the damage, which is stored on IPFS and notifies with the event "InspectionCompletedAndReported". 
The IPFShash is stored on the smart contract and the servicecount is incremented by 1 . Following the inspection, the vehicle insurer executes the validateClaim() function. If the claim matches the pre-agreed amount of insurance, the contract notifies the event ClaimValidationSuccessful and increments the successful claims by 1, else denyClaim() function are executed and all participants are notified with the message ClaimValidationDenied. All the transactions are recorded in the smart contract and are tamper-proof, preventing any fraudulent transaction or fake insurance claims. Once the claim is validated by the insurer successfully, the approvePayment () function is executed and the smart contract sends an event notification-PaymentApproved to all the active entities stating that the payment is approved which is presented in algorithm 3.

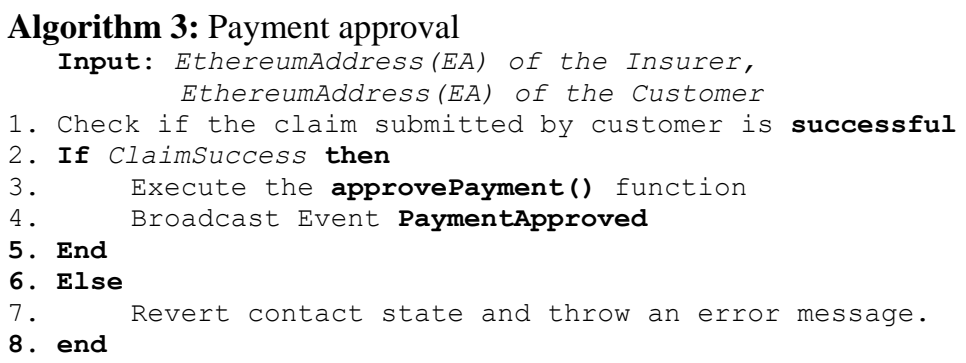

The smart contract was implemented on the Remix IDE-Ethereum platform and was coded in Solidity programming language. The fully functional code is available at https://github.com/NisharaNizamuddin/Auto_Insurance_BC. Figure 4 shows the constructor of the auto insurance smart contract. As shown in Figure 4, the customer creates the smart contract with the initial state of the smart contract being NotReady and the IPFShash of the report is "QmXgm5QVTy8pRtKrTPmoWPGXNesehCpP4jjFMTpVGamc1p", which remains to be a proof for the FIR registered for the damage caused. Other parameters such as numberOfRequestsByCustomer, numberOfApprovalsByAutoInsurer, numberOfValidationByGaragemember are also declared and initialised in the smart contract's constructor. The insurance amount is pre-determined, agreed, and is stored in the contract between the parties.

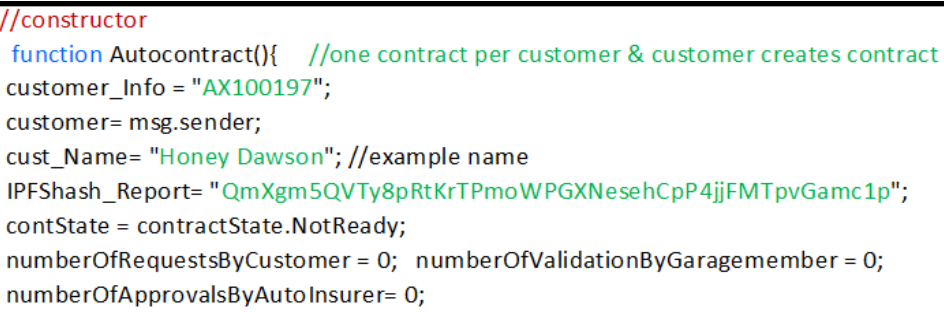

Figure 4. The constructor of the smart contract Autocontract ( $)^{1}$

The code snippet of the mapping to record the list of approvals and validation by the garage supervisor and insurance company are presented. Figure 5 shows mapping where every Ethereum address points to the address of the approved list of customers by the insurers and the garage supervisor's approval for payment processing.

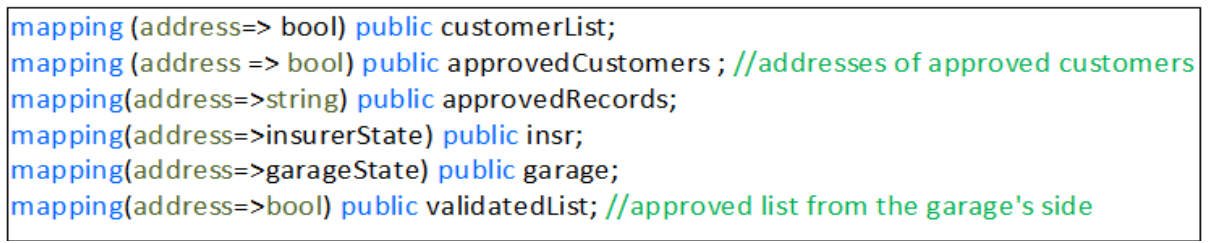

Figure 5. Mapping of customers claims approved by garage supervisor and insurance company ${ }^{1}$ 
The Ethereum addresses of various entities used for testing of contract are: policedept, insurance_company, customer, garage_supervisor: 0x14723A09ACff6D2A60DCdF7aA4AFf308FDDC160C, 0x4B0897b0513fdC7C541B6d9D7E929C4e5364D2dB, 0xCA35b7d915458EF540aDe6068dFe2F44E8fa733c, 0x583031D1113aD414F02576BD6afaBfb302140225 Respectively, Once the customer creates the contract and reports the event to the police department, the police personnel inspect and approve FIR. Next, Figure 6 illustrates the submitclaim () function, which is executed by the customer to approve the claims from the vehicle insurer. At this stage, the state of the smart contract is Inspected. The state of the contract changes to WaitingForClaimsApproval, and the insurer's state changes to SubmittedForApproval. The event Claimsubmitted is triggered. The customer waits for approval from the insurer who allocates the damage inspection task to a garage surveyor. The numberofRequestsByCustomer is incremented by 1 every time the customer submits an insurance claim request. Figures 6 and 7 shows the output logs for the function submitClaim() and event ClaimSubmitted broadcasted to the active entities in the contract.

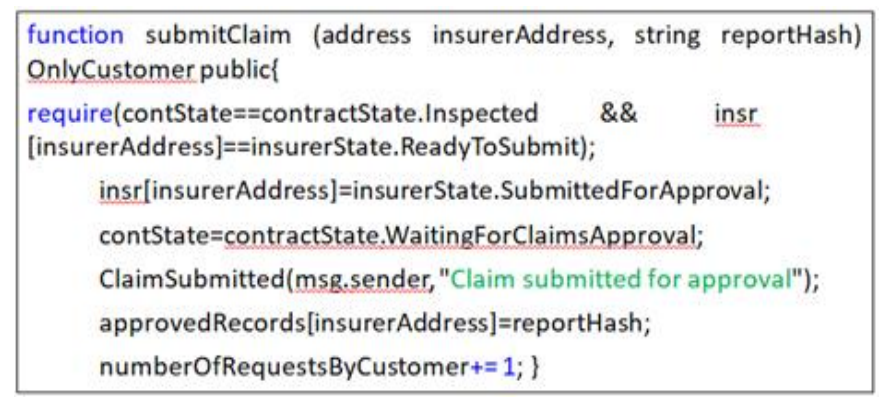

Figure 6. Customers submitting claim ${ }^{1}$

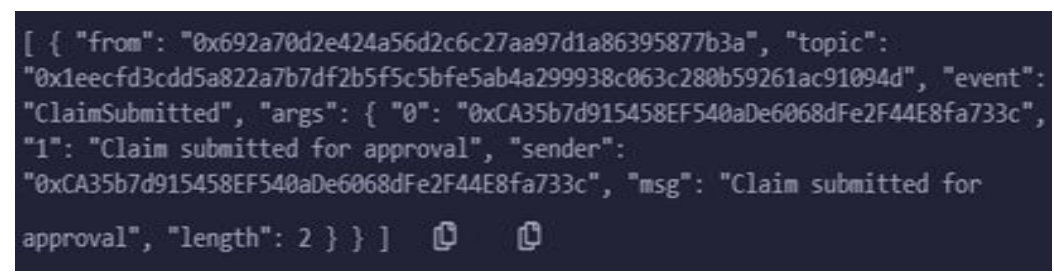

Figure 7. Event ClaimSubmitted ${ }^{1}$

Next, the Remix IDE console output for the appointment of the garage surveyor is shown in Figure 8. The function appointSurveyor () is executed by the insurance company. A modifier OnlyVehicleInsurer is used to restrict the access for the authorized entity alone, thereby preventing any malicious transaction. Further, the function takes the address of the customer claiming insurance as an input variable and sends a notification in the form of an event SurveyorAppointed broadcasting the message "Garage surveyor appointed to inspect damage" as shown in Figure 8.

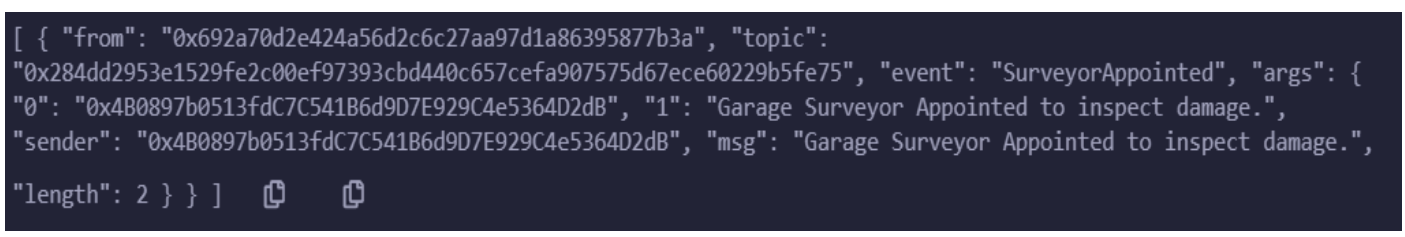

Figure 8. Logs showing appoint Surveyor ()$^{1}$ function

Finally, we present the output console for the inspectVehicle( ) function executed by garage supervisor in Figure 9. The original hash of the report stored on IPFS is QmXgm5QVTy8pRtKrTPmoWPGXNesehCpP4jjFMTpvGamc1p. The current state of the contract is 
SurveyorAppointed, and if the IPFShash of the insurance contract matches with the claim submitted by the consumer, and Insurer state changes to InspectionCompleted_claimsuccess and the numberofValidationByGaragemember is increased by 1 and the contract broadcasts the event ClaimvalidatedAndApproved as demonstrated in Figure 9. In case of a failed validation, the contract throws an error saying that the claim denied.

Finally, the logs showing payment approval followed by a successful inspection of the Vehicle is presented in Figure 10. Vehicle insurer executes approvePayment() where the contract state is GarageInspectionCompletedSuccess. Upon successful execution of function approvePayment (), the contract state changes to ProcessPayment, and the event PaymentApproved is notified with the message "insurance amount paid to customer". The smart contract ensures automatic payments, settlement of insurance claims, and transparent dispute settlements if any.

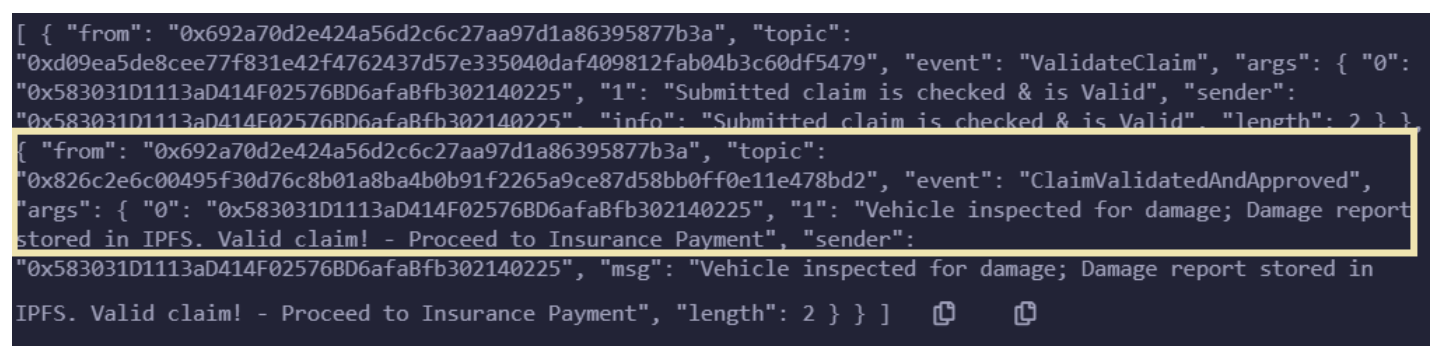

Figure 9. Logs showing inspectVehicle ( $)^{1}$ function

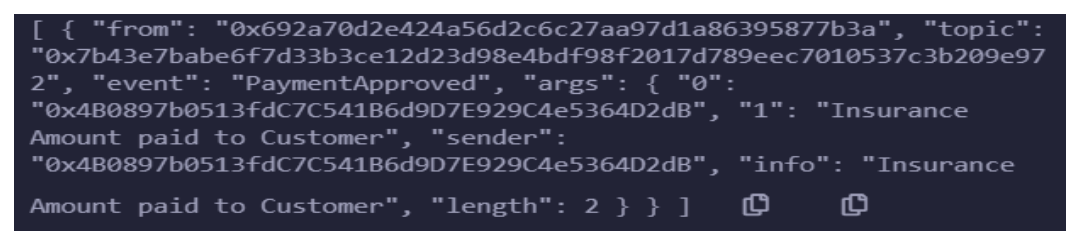

Figure 10. Logs showing approvePayment ( ) ${ }^{1}$ function

This proposed framework and algorithms will prove to be effective in terms of automatic insurance claim handling and dispute settlement between various stakeholders within the claim department as every transaction is recorded and is tamper-proof. Any kind of false amendment made to the surveyor's report or the initial agreement will modify the IPFShash which will in turn notify all the members that a fraudulent transaction has occurred and the smart contract reverts to its previous state. All transactions are recorded in the blockchain only when all the participating miner nodes agree with the outcome of it. This framework aims to improve the conventional auto-insurance sector's functioning by improving the security of transactions, safeguarding the identity of stakeholders, maintaining complete transparency, and facilitating automatic insurance claim/payment settlements.

\section{DISCUSSION}

The primary purpose of this study was to examine how blockchain technology and IPFS, when implemented, can benefit the auto-insurance sector. Earlier research suggests $[48,50,55]$ that blockchain can play a major role in auto-insurance document verification, document and identity management, and transfer of vehicle ownership. Further, other research studies clearly state the importance and role of decentralized blockchain technology $[17,21,73]$. Our results revealed that blockchain combined with the IPFS framework enhances the consumers' trust in the auto-insurance sector, improves the transparency of insurance transactions, and effective data and identity management of the insurance industry as a whole. It is evident that by using blockchain technology and IPFS framework all the documents and transactions are tamperproof, verifiable, and works on consensus protocol. In the longer run, blockchain technology can be combined with emerging technologies like artificial intelligence, edge computing, and machine learning. Stanciu [53] came up with a "blockchain-based distributed control systems for edge computing" which shows that blockchain juxtaposed with other emerging techniques and technologies has a promising future. 
The outcome of our research shows that any malicious attempt to change the records will result in a change in the IPFS hash which will notify all the miners about the malicious activity. If the miners don't approve the activity, then the transaction gets aborted and the block is not added to the blockchain.

Blockchain seems to be a disruptive and innovative technology that has gained importance across many platforms and many industries including healthcare, finance, robotics, food engineering have started designing platforms and modules for utilizing the benefits of this decentralized technology. It is certain to dominate the auto insurance sector with its disruptive properties. However, there are several implementation challenges to be examined before a large scale industry adoption is made possible. Various challenges to be tackled in implementing blockchain for the auto sector and social-autonomous vehicles in real-time are presented in this section. It is certain to dominate the auto insurance sector with its disruptive properties.

\subsection{Limitations}

However, there are several implementation challenges to be examined before a large-scale industry adoption is made possible [74]. Potential concerns associated with implementing blockchain technology for the auto-industry sector are presented in Figure 11. Various other challenges to be tackled in implementing blockchain for the auto sector and social-autonomous vehicles in real-time are presented in this section.

- Scalability: Blockchain suffers from scalability issues; with the increasing number of transactions the systems slow down and eventually become unsustainable [73, 75]. A transport ecosystem involves a large number of entities and transactions, and scalability remains a major issue.

- Lack of government standards: Government agencies are still analyzing the potential implications and benefits of blockchain technology.

- Regulations: Blockchain is still an emerging technology that makes it challenging to establish definite regulations and laws. In a blockchain network, there are multiple parties involved, located across the world, and every country and government has got different rules for various categories such as -individual privacy, legal procedures, liabilities, legal titles, and ownerships [75-77]. Blockchain, on the other hand, consumes a considerable amount of energy, as the miners use the computing power when adding new blocks of transaction data to the blockchain. New regulations have been proposed by world nations to check on the carbon emission and energy consumption which impacts the environment [78, 79].

- Usability issues: Blockchain technology is in its initial development stages, and most of the platforms face associated with blockchain face infrastructural issues [73]. The current tools used for development, testing, and evaluating the performance of methods have dependencies on third-party tools and technologies which raises questions on the decentralized features [73, 74].

- Security concerns: Smart contracts itself suffer from one of the major attacks-the DAO attack [80] which forced the developers and founders of Ethereum and the Bitcoin industry to reshape the security and robustness of smart contracts.

We have presented a decentralized blockchain-IPFS based framework in this paper. We aimed to present an elementary framework that can be used as a use case for the auto insurance sector using emerging blockchain technology. A similar model can be deployed for developing a transparent, secure, and tamperproof model for handling transactions in multiparty environments like the land registry, supply chain, logistics, healthcare, and education, to name a few.

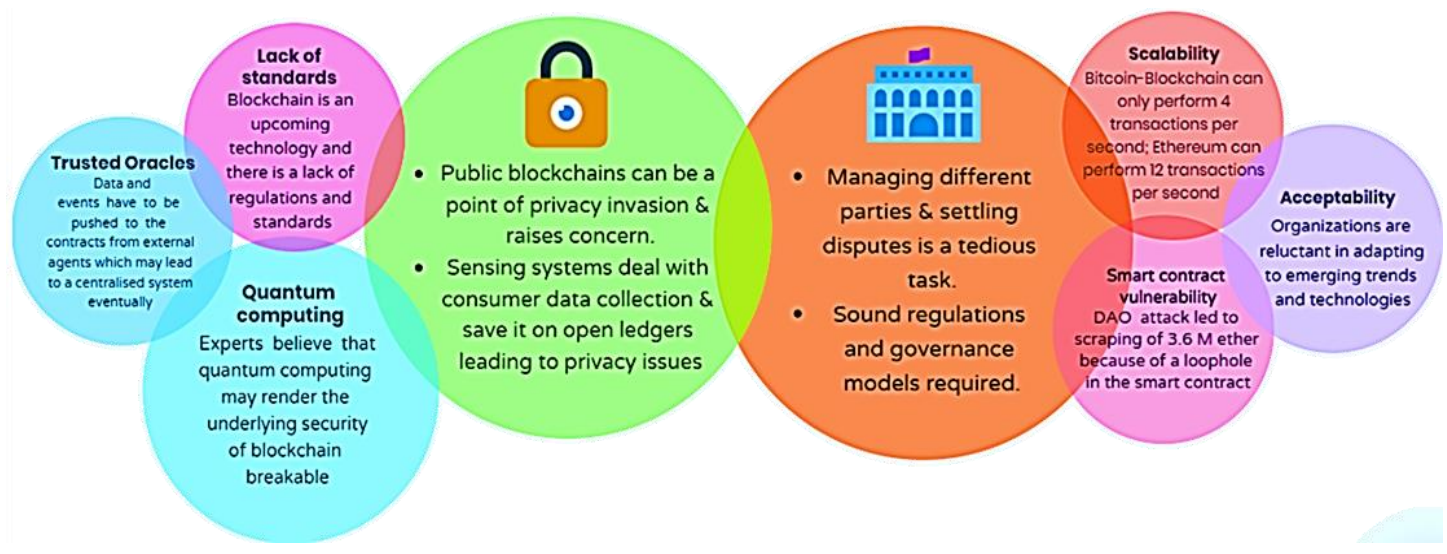

Figure 11. Potential challenges identified in implementing blockchain technology for the auto-insurance sector $[23,73-80,81]$ 


\section{CONCLUSION}

The automobile industry has embraced technological advances and major changes in terms of data management, collaboration, and information exchange since the time of the Industrial revolution. Blockchain technology has started to influence various business sectors including the government entities and corporate giants with its visionary properties. This article provides an insight into how blockchain technology can be incorporated to enhance the vehicular ecosystem and automotive sector. We presented an insight into blockchain-based applications and use cases for the transport ecosystem for data management, connected vehicle security, and infrastructure for the automobile industry. We proposed an Ethereum platform poweredblockchain-based decentralized auto-insurance framework that regulates the activities of the insurance sector, claims process in case of an accident or theft, and automates insurance payments. We emphasized the usage of IPFS for records management (claims record in this case) to store the record on a decentralized ledger, automate insurance payment claims, and avoid any error while handling the claims. In this article, we emphasize how blockchain-IPFS can be used together to augment overall institutional operations by improving the security and transparency of transactions. A similar framework can be used for improving transparency of transactions across various industries including land registry, supply chain industry, and logistics that aim to guide policymakers and government organizations. Further, as a part of future research work, we aim to implement the auto-insurance smart contract on the Hyperledger platform to facilitate and promote decentralized ledger technology as a part of smart city innovative investments.

\section{REFERENCES}

[1] J. Tropea, "Insurance disruption: How blockchain is transforming the industry," 2019. [Online]. Available: https://www.bankrate.com/insurance/blockchain-disruption/.

[2] A. Garg, J. Curtis, and H. Halper, "The financial impact of IT security breaches: what do investors think?" Journal Informations Systems Security, vol. 12, no. 1, pp. 22-33, 2003.

[3] A. Singh and K. B. Akhilesh, "The Insurance Industry-Cyber Security in the Hyper-Connected Age," in Smart Technologies, Springer, Singapore, pp. 201-219, 2020.

[4] R. Baheti and H. Gill, "Cyber-physical systems," The impact of control technology, vol. 12, no. 1, pp. 161-166, 2011.

[5] Frost and Sullivan, "Mobility: Automotive \& Transportation," 2019. [Online]. Available: https://ww2.frost.com/research/industry/mobility-automotivetransportation/.

[6] R. C. Witt, "Pricing problems in automobile insurance: An economic analysis," Journal of Risk and Insurance, pp. 75-93, 1973.

[7] S. Tennyson, "Challenges and approaches to consumer protection in the insurance industry," The Future of Insurance Regulation and Supervision: A Global Perspective, pp. 191-205, 2011.

[8] R. K. Goel, "Insurance fraud and corruption in the United States," Applied Financial Economics, vol. 24, no. 4, pp. 241-246, 2014.

[9] J. E. Nicholson, "Challenges for the Insurance Industry in the Future," Journal of Insurance Regulation, vol. 38, no. 6, 2019.

[10] M. Swan, "Anticipating the economic benefits of blockchain," Technology innovation management review, vol. 7, no. 10, pp. 6-13, 2017.

[11] M. Eling and M. Luhnen, "Efficiency in the international insurance industry: A cross-country comparison," Journal of Banking \& Finance, vol. 34, no. 7, pp. 1497-1509, 2010.

[12] M. F. Grace and R. W. Klein, "The future of insurance regulation in the United States," Brookings Institution Press, 2009.

[13] D. I. Tselentis, G. Yannis, and E. I. Vlahogianni, "Innovative motor insurance schemes: A review of current practices and emerging challenges," Accident Analysis \& Prevention, vol. 98, pp. 139-148, 2017.

[14] N. P. Katz, et al., "Prescription opioid abuse: challenges and opportunities for payers," The American journal of managed care, vol. 19, no. 4, p. 295, 2013.

[15] Acko, "5 Common Problems Faced by Vehicle Insurance Claims Team," [Online]. Available: https://www.acko.com/articles/general-info/.

[16] P. Gao, R. Hensley, and A. Zielke, "A road map to the future for the auto industry," McKinsey Quarterly, pp. 1-11, 2014.

[17] S. Viaene and G. Dedene, "Insurance fraud: issues and challenges," The Geneva Papers on Risk and InsuranceIssues and Practice, vol. 29, no. 2, pp. 313-333, 2004.

[18] P. Coccorese, "Information sharing, market competition, and antitrust intervention: a lesson from the Italian insurance sector," Applied Economics, vol. 44, no. 3, pp. 351-359, 2012.

[19] S. Daley, Nine companies using blockchain to revolutionize insurance, 2020. [Online]. Available: https://builtin.com/blockchain/blockchain-insurancecompanies.

[20] J. Singh, "Identity fraud's impact on the insurance sector," Thomson Reuters. [Online]. Available: https://legal.thomsonreuters.com/en/insights/articles/identity-frauds-impact-on-the-insurance-sector.

[21] S. Nagrecha, R. A. Johnson, and N. V. Chawla, "FraudBuster: Reducing fraud in an auto insurance market," Big data, vol. 6, no. 1, pp. 3-12, 2018.

[22] A. Chehri and H. T. Mouftah, "Autonomous vehicles in the sustainable cities, the beginning of a green adventure," Sustainable Cities and Society, vol. 51, p. 101751, 2019. 
[23] J. Chen, et al., “A Blockchain-Driven Supply Chain Finance Application for Auto Retail Industry,” Entropy, vol. 22, no. 1, p. 95, 2020.

[24] R. Roriz and J. L. Pereira, "Avoiding Insurance Fraud: A Blockchain-based Solution for the Vehicle Sector," Procedia Computer Science, vol. 164, pp. 211-218, 2019.

[25] S. Terzi, et al., "Blockchain 3.0 Smart Contracts in E-Government 3.0 Applications," arXiv preprint arXiv:1910.06092, 2019.

[26] S. Abdallah, N. Nizamuddin, and A. Khalil, "Blockchain for improved safety of smart buildings," in International Conference Connected Smart Cities 2019, Portugal, 2019.

[27] S. Nakamoto, "A peer-to-peer electronic cash system," Bitcoin, 2008. [Online]. Available: http://bitcoin.org/Bitcoin.pdf.

[28] M. Eling and M. Luhnen, "Efficiency in the international insurance industry: A cross-country comparison," Journal of Banking \& Finance, vol. 34, no. 7, pp. 1497-1509, 2010.

[29] M. F. Hsieh, C. C. Lee, and S. J. Yang, "The Impact of Diversification on Performance in the Insurance Industry: The Roles of Globalisation, Financial Reforms, and Global Crisis," The Geneva Papers on Risk and InsuranceIssues and Practice, vol. 40, no. 4, pp. 585-631, 2015.

[30] M. Demir, O. Turetken, and A. Ferworn, "Blockchain-Based Transparent Vehicle Insurance Management," in 2019 Sixth International Conference on Software Defined Systems (SDS) IEEE, 2019, pp. 213-220.

[31] E. R. W. Peterson, et al., "The future of mobility and shifting risk-AIG the future of mobility and shifting risk and the data," AIG-Innovation-Part 8 in a Series. [Online]. Available: https://vdocuments.mx/document/the-future-ofmobility-and-shifting-risk-aig-the-future-of-mobility-and-shifting.html.

[32] "Insurance issues we are exploring," $P w C$ USA. [Online]. Available: https://www.pwc.com/us/en/industries/insurance/library/top-issues.html.

[33] Digital UAE, Blockchain in the UAE government, 2019. [Online]. Available: https://government.ae/en/about-theuae/digitaluae/blockchain-in-the-uae-government.

[34] "KBC Group: KBC launches pilot project to test the authenticity of KBC documents," Market Screener, 2017. [Online]. Available: https://www.marketscreener.com/quote/stock/KBC-GROUPE-SA-5967/news/KBC-GroupKBC-launches-pilot-project-to-test-authenticity-of-KBC-documents-24642688/.

[35] J. Benet, "IPFS-content addressed, versioned, p2p file system," arXiv preprint arXiv: 1407.3561, 2014.

[36] J. Singh, "Identity fraud's impact on the insurance sector," Thomson Reuters. [Online]. Available: https://legal.thomsonreuters.com/en/insights/articles/identity-frauds-impact-on-the-insurance-sector.

[37] "Reports, and Publication: Insurance Fraud," FBI, [Online]. Available: https://www.fbi.gov/statsservices/publications/insurance-fraud.

[38] S. Viaene and G. Dedene, "Insurance fraud: issues and challenges," The Geneva Papers on Risk and InsuranceIssues and Practice, vol. 29, no. 2, pp. 313-333, 2004.

[39] R. Tillman, "Abandoned Consumers: Deregulation and Fraud in the California Auto Insurance Industry," Social Policy and Society, vol. 2, no. 1, pp. 45-53, 2003.

[40] A. Dorri, M. Steger, S. S. Kanhere, and R. Jurdak, "Blockchain: A distributed solution to automotive security and privacy," IEEE Communications Magazine, vol. 55, no. 12, pp. 119-125, 2017.

[41] Z. Yang, K. Zheng, K. Yang, and V. C. Leung, "A blockchain-based reputation system for data credibility assessment in vehicular networks," in 2017 IEEE 28th annual international symposium on personal, indoor, and mobile radio communications (PIMRC), Canada, Oct. 2017, pp. 1-5.

[42] L. Li, J. Liu, L. Cheng, S. Qiu, W. Wang, X. Zhang, and Z. Zhang, "Creditcoin: A privacy-preserving blockchainbased incentive announcement network for communications of smart vehicles," IEEE Transactions on Intelligent Transportation Systems, vol. 19, no. 7, pp. 2204-2220, 2018.

[43] F. Gao, L. Zhu, M. Shen, K. Sharif, Z. Wan, and K. Ren, "A blockchain-based privacy-preserving payment mechanism for vehicle-to-grid networks," IEEE Network, vol. 32, no. 6, pp. 184-192, 2018.

[44] C. Oham, R. Jurdak, S. S. Kanhere, A. Dorri, and S. Jha, "B-fica: Blockchain-based framework for auto-insurance claim and adjudication," in 2018 IEEE International Conference on Internet of Things (iThings) and IEEE Green Computing and Communications (GreenCom) and IEEE Cyber, Physical and Social Computing (CPSCom) and IEEE Smart Data (SmartData) IEEE, 2018, pp. 1171-1180.

[45] Viereckl, et al., "Digital Auto Report 2019-The future is here: flexible carmakers must balance metal and mobility," Strategy \& Part of PwC network, 2018. [Online]. Available: https://www.strategyand.pwc.com/de/studie/future-ishere.

[46] H. T. Vo, L. Mehedy, M. Mohania, and E. Abebe, "Blockchain-based data management and analytics for microinsurance applications," in Proceedings of the 2017 ACM on Conference on Information and Knowledge Management, 2017, pp. 2539-2542.

[47] S. Chekriy and Y. Mukhin, "Blockchain Platform for Insurance-related Products," Glass Cube, pp. 1-33, 2018.

[48] P. Fraga-Lamas and T. M. Fernández-Caramés, "A review on blockchain technologies for an advanced and cyberresilient automotive industry," IEEE Access, vol. 7, pp. 17578-17598, 2019.

[49] European Start-up Prize for Mobility, "How blockchain will revolutionize the way we use cars," 2018. [Online]. Available: https://startupprize.eu/blockchain-will-revolutionise-way-use-cars/.

[50] "Renault embraces blockchain to track vehicle maintenance history," E \& T Engineering and Technology, 2017. [Online]. Available: https://eandt.theiet.org/content/articles/2017/07/renaultembraces-blockchain-to-track-vehiclemaintenance-history/. 
[51] K. P. M. L. P. Weerasinghe and M. C. Wijegunasekara, "A comparative study of data mining algorithms in the prediction of auto insurance claims," European International Journal of Science and Technology, vol. 5, no. 1, pp. 47-54, 2016.

[52] J. Song, et al., "Physical Data Auditing for Attack Detection in Cyber-Manufacturing Systems: Blockchain for Machine Learning Process," ASME 2019 International Mechanical Engineering Congress and Exposition, 2019.

[53] A. Stanciu, "Blockchain-based distributed control system for edge computing," 201721 st International Conference on Control Systems and Computer Science (CSCS), Bucharest, 2017, pp. 667-671.

[54] I. J. Jensen, D. F. Selvaraj, and P. Ranganathan, "Blockchain Technology for Networked Swarms of Unmanned Aerial Vehicles (UAVs)," 2019 IEEE 20th International Symposium on "A World of Wireless, Mobile and Multimedia Networks" (WoWMoM), Washington, DC, USA, 2019, pp. 1-7.

[55] V. Gatteschi, F. Lamberti, and C. Demartini, "Blockchain Technology Use Cases", In Advanced Applications of Blockchain Technology, Springer, Singapore, pp. 91-114, 2020.

[56] Y. Yahiatene and A. Rachedi, "Towards a Blockchain and Software-Defined Vehicular Networks Approaches to Secure Vehicular Social Network," 2018 IEEE Conference on Standards for Communications and Networking (CSCN), Paris, 2018, pp. 1-7.

[57] Z. Su, Y. Wang, Q. Xu, M. Fei, Y. C. Tian, and N. Zhang, "A secure charging scheme for electric vehicles with smart communities in energy blockchain," IEEE Internet of Things Journal, vol. 6, no. 3, pp. 4601-4613, 2018.

[58] S. Aggarwal, R. Chaudhary, G. S. Aujla, N. Kumar, K. K. R. Choo, and A. Y. Zomaya, "Blockchain for smart communities: Applications, challenges, and opportunities," Journal of Network and Computer Applications, vol. 144, pp. 13-48, 2019.

[59] G. S. Aujla, M. Singh, A. Bose, N. Kumar, G. Han, and R. Buyya, "BlockSDN: Blockchain-as-a-Service for Software-Defined Networking in Smart City Applications," IEEE Network, vol. 34, no. 2, pp. 83-91, 2020.

[60] "Daring to be first-How auto pioneers are taking the plunge into the blockchain," IBM Institute for Business Value, [Online]. Available: https://public.dhe.ibm.com/common/ssi/ecm/29/en/29022329usen/29022329usen00_29022329USEN.pdf.

[61] M. Montecchi, K. Plangger, and M. Etter, "It's real, trust me! Establishing supply chain provenance using blockchain," Business Horizons, vol. 62, no. 3, pp. 283-293, 2019.

[62] A. Banerjee, "Blockchain technology: supply chain insights from ERP," in Advances in Computers, vol. 111, pp. 69-98, 2018.

[63] L. Ajao, "A Secure Tracking Automobile System for Oil and Gas Distribution using Telematics and Blockchain Techniques," Balkan Journal of Electrical and Computer Engineering, vol. 7, no. 3, pp. 257-268, 2019.

[64] S. A. Abeyratne and R. P. Monfared, "Blockchain ready manufacturing supply chain using a distributed ledger," International Journal of Research in Engineering and Technology, vol. 5, no. 9, pp. 1-10, 2016.

[65] T. Reimers, F. Leber, and U. Lechner, "Integration of Blockchain and the Internet of Things in a Car Supply Chain," 2019 IEEE International Conference on Decentralized Applications and Infrastructures (DAPPCON), Newark, CA, USA, 2019, pp. 146-151.

[66] Y. Balasubramaniam and P. S. V. Sathyanarayanan, "Enhancing Connected Vehicle Security with Blockchain," International Journal of Advances in Scientific Research and Engineering (IJASRE), vol. 4, no. 8, pp. 189-193, 2018.

[67] M. Cebe, et al., "Block4forensic: An integrated lightweight blockchain framework for forensics applications of connected vehicles," IEEE Communications Magazine, vol. 56, no. 10, pp. 50-57, 2018.

[68] J. Turato, "Blockchain-based connected user communication and interface system," U.S. Patent Application 16/221, p. 491, 2019.

[69] V. Sharma, "Blockchain-Its Role in Demand and Supply Planning," The Journal of Business Forecasting, vol. 38, no. 4, pp. 9-11, 2019.

[70] D. M. West, "Moving forward: self-driving vehicles in China, Europe, Japan, Korea, and the United States," Center for Technology Innovation at Brookings: Washington, DC, USA, 2016.

[71] K. L. Brousmiche, T. Heno, C. Poulain, A. Dalmieres, and E. B. Hamida, "Digitizing, securing, and sharing vehicles life-cycle over a consortium blockchain: Lessons learned," in 2018 9th IFIP international conference on new technologies, mobility, and security (NTMS), 2018, pp. 1-5.

[72] A. Ebrahimi, "Identity management service using a blockchain providing certifying transactions between devices," U.S. Patent 9,722,790, 2017.

[73] I. C. Lin and T. C. Liao, "A survey of blockchain security issues and challenges," International Journal of Network Security, vol. 19, no. 5, pp. 653-659, 2017.

[74] L. Hughes, et al., "Blockchain research, practice, and policy: Applications, benefits, limitations, emerging research themes and research agenda," International Journal of Information Management, vol. 49, pp. 114-129, 2019.

[75] "Key challenges," $\quad$ Deloitte, $2019 . \quad$ [Online]. https://www2.deloitte.com/content/dam/Deloitte/uk/Documents/Innovation/deloitte-uk-blockchain-keychallenges.pdf.

[76] J. Sebastian, "Blockchain in financial services: Regulatory landscape and future challenges," 2016. [Online]. Available: https://www.bbvaresearch.com/wp-content/uploads/2016/12/WP_16-20.pdf 
[77] P. Yeoh, "Regulatory issues in blockchain technology," Journal of Financial Regulation and Compliance, vol. 25, no. 2, pp. 196-208, 2017.

[78] J. Truby, "Decarbonizing Bitcoin: Law and policy choices for reducing the energy consumption of Blockchain technologies and digital currencies," Energy research \& social science, vol. 44, pp. 399-410, 2018.

[79] Y. Lu, "The blockchain: State-of-the-art and research challenges," Journal of Industrial Information Integration, vol. 15, pp. 80-90, 2019.

[80] M. I. Mehar, et al., "Understanding a revolutionary and flawed grand experiment in the blockchain: the DAO attack," Journal of Cases on Information Technology (JCIT), vol. 21, no. 1, pp. 19-32, 2019.

[81] W. Mougayar, "The business blockchain: promise, practice, and application of the next Internet technology," John Wiley \& Sons, 2016.

\section{BIOGRAPHIES OF AUTHORS}

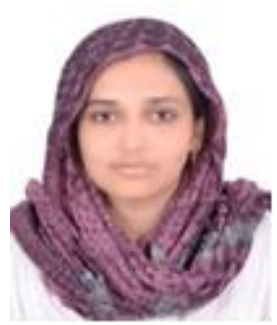

N. Nizamuddin is a Research \& Teaching Assistant in the College of Technological Innovation at Zayed University. She received her Master's degree in Computer Science and Engineering from VIT University, India. Before joining Zayed University, Ms. Nishara was working as a Researcher at Khalifa University and Abu Dhabi University, UAE and as Software Engineer at BOSCH. Her research interest includes Blockchain, Distributed Systems, Cloud Computing, and Machine Learning \& is currently working on Blockchain projects related to Healthcare and Food security.

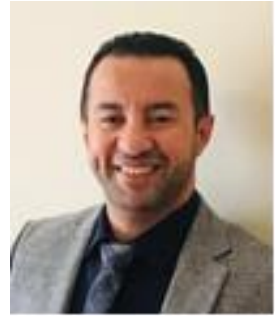

A. Abugabah is an Assistant Professor at the College of Technological Innovation at Zayed University. He worked in higher education in Australia where he received his degrees in information systems. Dr. Abugabah also worked in the airline industry in the Aircraft Engineering and Supply Chain management. Before Joining Zayed University in 2016, Dr. Abugabah was involved in administration as an Associate Dean and a University Council Member at the American University, UAE. His research interests include Information Systems, Enterprise Applications, and Development, Healthcare Information Systems, and RFID in Healthcare. 